\title{
In situ evidence of breaking the ion frozen-in condition via the non-gyrotropic pressure effect in magnetic reconnection
}

\author{
L. Dai ${ }^{1,2}$, C. Wang ${ }^{1}$, V. Angelopoulos ${ }^{3}$, and K.-H. Glassmeier ${ }^{4}$ \\ ${ }^{1}$ State Key Laboratory of Space Weather, National Space Science Center, Chinese Academy of Sciences, Beijing, China \\ ${ }^{2}$ School of Physics and Astronomy, University of Minnesota, Minneapolis, MN, USA \\ ${ }^{3}$ Department of Earth, Planetary and Space Sciences and Institute of Geophysics and Planetary Physics, \\ University of California, Los Angeles, CA, USA \\ ${ }^{4}$ Institute of Geophysics and extraterrestrial Physics, University of Braunschweig, Braunschweig, Germany \\ Correspondence to: L. Dai (ldai@ spaceweather.ac.cn)
}

Received: 13 July 2015 - Revised: 12 August 2015 - Accepted: 3 September 2015 - Published: 17 September 2015

\begin{abstract}
For magnetic reconnection to proceed, the frozenin condition for both ion fluid and electron fluid in a localized diffusion region must be violated by inertial effects, thermal pressure effects, or inter-species collisions. It has been unclear which underlying effects unfreeze ion fluid in the diffusion region. By analyzing in situ THEMIS (Time History of Events and Macroscale Interactions during Substorms) spacecraft measurements at the dayside magnetopause, we present clear evidence that the off-diagonal components of the ion pressure tensor is mainly responsible for breaking the ion frozen-in condition in reconnection. The off-diagonal pressure tensor, which corresponds to a non-gyrotropic pressure effect in this event, is a fluid manifestation of ion demagnetization in the diffusion region. From the perspective of the ion momentum equation, the reported non-gyrotropic ion pressure tensor is a fundamental aspect in specifying the reconnection electric field that controls how quickly reconnection proceeds.
\end{abstract}

Keywords. Space plasma physics (magnetic reconnection)

\section{Introduction}

Magnetic reconnection is considered to drive global-scale dynamics in Earth's magnetosphere (Angelopoulos et al., 2008, 2013), solar flares (Parker, 1957; Petschek, 1964), laboratory plasmas and astrophysical systems. Magnetic reconnection changes the magnetic field topology and releases magnetic energy into particle energy in plasmas. As reconnection occurs, magnetic field lines appear to "break" and "reconnect" at the $X$-line (Fig. 1a). Outside the diffusion region, plasma motions are frozen to magnetic field lines that behaves like elastic strings. Within the diffusion region, the violation of plasma that is frozen-in allows magnetic field lines to disconnect and reconnect.

The name "diffusion region" originates from the early Sweet-Parker reconnection model, in which oppositely directed magnetic field lines in the current sheet diffuse into the plasma as plasmas are demagnetized by inter-species collisions (Parker, 1957; Petschek, 1964). The term diffusion region has been extended to include any physical process that can violate the frozen-in condition (Vasyliunas, 1975; Hesse et al., 1999). The physics in the limited diffusion region is of high importance in magnetic reconnection. The global-scale evolution of the magnetic topology relies on the reconfiguration of magnetic field lines in the diffusion region. The effects responsible for breaking the frozen-in condition specify the reconnection electric field that controls how quickly reconnection proceeds.

For reconnection to proceed, the frozen-in condition for both ion and electron must be violated in the diffusion region. In the case of only one species being unfrozen from the field line, one can trace the frozen magnetic field lines tied to the other magnetized species. The exact condition for unfreezing ion fluid is a non-zero curl of $\boldsymbol{E}+\boldsymbol{v}_{\mathrm{i}} \times \boldsymbol{B}$ based on the frozenin theorem. According to the ion fluid momentum equation without approximation,

$$
\boldsymbol{E}+\boldsymbol{v}_{\mathrm{i}} \times \boldsymbol{B}=\left(1 / n_{\mathrm{i}} q_{\mathrm{i}}\right) \nabla \cdot \mathbf{P}_{\mathrm{i}}+\left(m_{\mathrm{i}} / q_{\mathrm{i}}\right) \mathrm{d} \boldsymbol{v}_{\mathrm{i}} / \mathrm{d} t+\left(m_{\mathrm{i}} / q_{\mathrm{i}}\right) v_{\mathrm{ie}}\left(\boldsymbol{v}_{\mathrm{i}}-\boldsymbol{v}_{\mathrm{e}}\right),
$$


where $\boldsymbol{E}$ is the electric field, $\mathbf{P}_{\mathrm{i}}$ is the ion pressure tensor, $\boldsymbol{v}_{\mathrm{i}}\left(\boldsymbol{v}_{\mathrm{e}}\right)$ is the ion (electron) flow velocity, and $v_{\mathrm{ie}}$ is the effective ion-electron collision frequency. As evident from the ion momentum Eq. (1), the ion frozen-in condition can be violated by three nonideal effects, i.e., an anisotropic ion pressure tensor, an ion inertial (acceleration) effect or interspecies collisions manifested as friction. These nonideal effects are the fluid manifestation of the kinetic effects leading to ion demagnetization in reconnection. The expression (1) and arguments for ions apply equally to electrons after a suitable change of charge, mass and subindexes.

The main nonideal effects for ion and electron fluid in reconnection are still debated. Reconnection models are categorized according to which nonideal process is thought to be dominant. In the resistive reconnection model, inter-species collision is the dominant effect. The collisions can be either binary or anomalous (induced by wave-particle interactions that are not clearly understood). In collisionless reconnection models where effective ion-electron collisions are infrequent, the nonideal effects have to be anisotropic pressure effects and/or inertial effects. As plasmas are convected toward the reconnection site, ions first become demagnetized at the characteristic scale length of ion motions, while electrons are still magnetized (Sonnerup, 1979). Electrons are expected to be demagnetized on a smaller spatial scale.

The reconnection electric field, defined in the co-moving frame of the current layer, controls how quickly (by $\boldsymbol{E} \cdot \boldsymbol{J}$ ) magnetic energy is converted to particle energy in reconnection. The reconnection electric field is the same in the ion momentum (Eq. 1) and the electron momentum equation. From the perspective of the ions (electrons), the reconnection electric field is the sum of the nonideal electric field supported by ion (electron) nonideal effects and the simple ion (electron) convection electric field. Although demagnetization is more difficult for electrons, the reconnection electric field is equally explainable in terms of ions or electrons near the $X$ line (Hesse et al., 2011; Cai and Lee, 1997). In this sense, ion demagnetization and electron demagnetization are two different but equally fundamental aspects in specifying the reconnection electric field.

In collisionless reconnection, ion decoupling from the magnetic field lines is considered to produce a Hall effect (Sonnerup, 1979; Deng and Matsumoto, 2001; Nagai et al., 2001; Øieroset et al., 2001), but what nonideal effect is responsible for unfreezing ion fluid in the first place has not been clear. Intuitively, it is tempting to attribute ion decoupling to the Hall current term in the generalized Ohm's law, $\boldsymbol{E}+\boldsymbol{v}_{\mathrm{i}} \times \boldsymbol{B}=(1 / n q) \boldsymbol{J} \times \boldsymbol{B}$, but this relation is a trivial equivalence of the electron frozen-in condition $\left(\boldsymbol{E}+\boldsymbol{v}_{\mathrm{e}} \times \boldsymbol{B}=0\right)$. Ion information is canceled out from this relation and nothing specific about nonideal effects of ions can be inferred. Studies of ion-scale physics in reconnection have been usually guided by the generalized Ohm's law, which is essentially a combination of electron and ion momentum equations with some approximations. Although appropriate for electrons to a certain extent, these approximations neglect and hide important aspects of ion dynamics. The correct approach to investigate ion-scale physics is the full momentum equation without approximation (Hesse et al., 1999, 2011; Cai and Lee, 1997).

Experimental clarification of the ion nonideal effects requires a comparison between the nonideal electric field $(\boldsymbol{E}+$ $\left.\boldsymbol{v}_{\mathrm{i}} \times \boldsymbol{B}\right)$ and ion pressure and/or inertial terms. Although attempts have been made to compare the nonideal reconnection electric field with the divergence of the electron pressure tensor, the electron diffusion region was too small for the spacecraft to encounter (Henderson et al., 2006). On the ion skin depth scale, past spacecraft observations have reported ion kinetic features such as non-gyrotropic ions and counterstreaming ions during reconnection (Hoshino et al., 1998; Nagai et al., 1998; Wygant et al., 2005; Zhou et al., 2009; Aunai et al., 2011), but how these effects are linked to ion demagnetization and the violation of the ion frozen-in condition has been elusive. Here we report observations from an encounter of the THEMIS (Time History of Events and Macroscale Interactions during Substorms) spacecraft (Angelopoulos, 2008; Sibeck and Angelopoulos, 2008) with the diffusion region near the reconnection $X$-line at the Earth's magnetopause. The comprehensive field instrumentation and measurements of ion velocity distribution (Bonnell et al., 2008; McFadden et al., 2008) on the THEMIS spacecraft provide an ideal opportunity to address the question of ion demagnetization in reconnection. By comparing the nonideal reconnection electric field with ion pressure and/or inertial terms, we for the first time identify the nonideal effects corresponding to ion demagnetization in the diffusion region.

\section{Data analysis}

On 13 February 2013, THEMIS spacecraft E moved into the magnetopause and detected a reconnection diffusion region (see Fig. 1b). THEMIS E was at $(6.1,-6.9,-0.6)$ in units of Re (Earth radii) in the geocentric solar magnetospheric coordinate system (GSM). We adopted the boundary normal coordinate NML to study the magnetopause current sheet. $\mathbf{N}$, $(-0.78,0.59,0.21) \mathrm{GSM}$, is the boundary normal direction (outward) as determined by the minimal variance direction of the four samples per second magnetic fields from 23:24:20 to 23:25:20 UT; $\mathbf{L},(-0.07,-0.42,0.90)$, GSM is the direction of maximum variance of the magnetic field, and $\mathbf{M}$ completes the right-hand orthogonal coordinate, directed out of plane. The corresponding eigenvalues for the eigenvectors are 9.4, 18.7 and 3011.4. There is some uncertainty in $\mathbf{M}$ and $\mathbf{N}$ since the ratio of the two corresponding eigenvalues is less than 3 , but the $\mathbf{N}$ from minimal variance analysis (MVA) varies little if we shift the interval by $10 \mathrm{~s}$. The determined $\mathbf{N}$ direction is also very close to the $\mathbf{N}(-0.90,0.42,0.10)$ obtained from a longer interval (23:23:00 to 23:33:00 UT). These facts suggest that the $N$ direction from MVA is reasonably good. 
a)

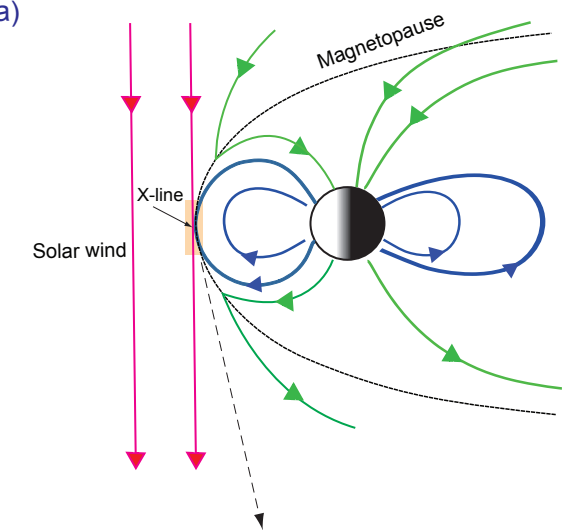

b)

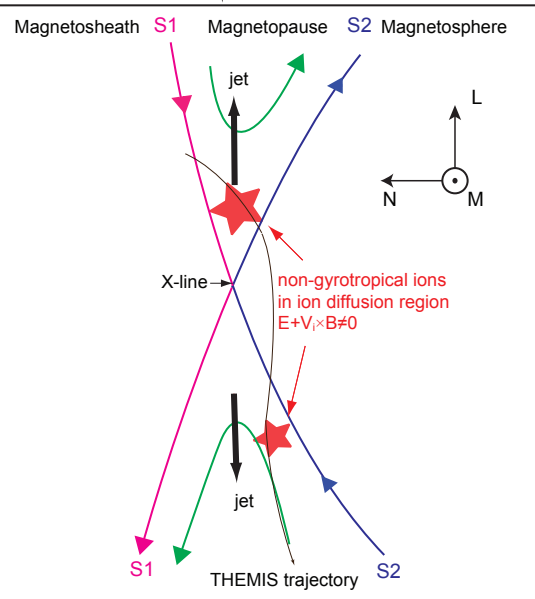

Figure 1. Schematics of magnetic reconnection in the Earth's magnetopause. Panel (a): the magnetic field geometry at the dayside magnetopause reconnection viewed in the noon-midnight plane. Magnetic field lines near magnetopause reconnection can be divided into three classes according to their topology: (1) interplanetary field lines (red) with no magnetic foot on the Earth; (2) "open" field lines (green) with one magnetic foot connected to the Earth and (3) "closed" field lines (blue) with both magnetic feet connected to the Earth. Two branches of separatrix surface S1 and S2 intersect along a magnetic " $X$-line" directed out of the plane. Panel (b): the expanded view of the region surrounding the $X$-line. A portion of the plasma outflow came across the ion diffusion region identified by a significant deviation of $\boldsymbol{E}$ from $-\boldsymbol{V}_{\mathbf{i}} \times \boldsymbol{B}$.

Figure 2 shows measurements of fields and particles during the magnetopause crossing. The magnetopause current sheet, indicated by a change in $\boldsymbol{B}_{L}$ from -40 to $60 \mathrm{nT}$, was observed from 23:24:40 to 23:25:00 UT. Northward plasma flow with velocities as large as $130 \mathrm{~km} \mathrm{~s}^{-1}$ was detected from 23:24:42 to $23: 25: 05 \mathrm{UT}$, immediately after the spacecraft crossed the separatrix S1 at 23:24:40 UT. The separatrix S1 is identified by a sudden increase of $1-10 \mathrm{keV}$ electrons that originated in the magnetosphere. The electron characteristics are a good indication of separatrix field lines because of their small gyroradius and large mobility along the magnetic field. Around 23:25:10 UT, THEMIS E crossed the separa- trix S2 and moved into the magnetosphere. The separatrix S2 is identified by a boundary between a broad spectrum of mixed electrons and a dominant magnetosphere electrons. Between 23:25:10 and 23:25:30 UT, the spacecraft was away from the current sheet and the diffusion region, as evidenced by the large $\boldsymbol{B}_{L}$ component. After 23:25:30 UT, THEMIS E moved back into the magnetopause and observed the southward plasma flow. $\boldsymbol{B}_{N}$ shows a variation of a few nanotesla on a $20 \mathrm{~s}$ timescale in addition to the direct current (DC) component near the current sheet. This variation in $\boldsymbol{B}_{N}$ may result from an eigenmode of the current sheet surface waves (Dai, 2009; Dai et al., 2011). The DC $\boldsymbol{B}_{N}$ is negative in the northward plasma flow and positive in the southward flow, consistent with the prediction that the bidirectional plasma flows are accelerated by a $\boldsymbol{J} \times \boldsymbol{B}_{N}$ slingshot effect resulting from reconnection. The reversal of the ion flow is correlated with the reversal of $\boldsymbol{B}_{N}$, suggesting that the determination of $\mathbf{N}$ is reasonably good in this event. The observed bidirectional reconnection outflows indicate that the spacecraft moved from the northward side to the southward side of an active reconnection $X$-line. THEMIS E did not encounter the $X$-line $\left(\boldsymbol{B}_{N}=0, \boldsymbol{B}_{L}=0\right)$ but its surrounding region.

Figure $2 \mathrm{~g}$ exhibits a significant deviation in the measured electric field $\boldsymbol{E}_{\mathrm{M}}$ from $-\boldsymbol{v}_{\mathrm{i}} \times \boldsymbol{B}$ from 23:24:44 UT to 23:24:58 UT during the current sheet crossing. This deviation illustrates the violation of the ion frozen-in condition as a support of the reconnection electric field $\boldsymbol{E}_{\mathrm{M}}$, experimentally defining the so-called ion diffusion region. The period from $23: 25: 20$ to $23: 25: 44$ UT is the edge of the diffusion region and far from the current sheet center. The ion diffusion region in the current sheet is marked by a pink rectangle. Within the diffusion region, a remarkable normal electric field $\boldsymbol{E}_{N}$ as large as $10 \mathrm{mV} \mathrm{m}^{-1}$ was observed. The out-of-plane magnetic field $\boldsymbol{B}_{M}$ displayed one bump around the current sheet center $\left(\boldsymbol{B}_{L} \sim 0\right)$; it was about $10 \mathrm{nT}$ larger than the average guide field outside the current sheet. The location and waveforms of $\boldsymbol{E}_{N}$ and $\boldsymbol{B}_{M}$ are completely consistent with those of the Hall magnetic fields and electric fields in the asymmetric reconnection reported in early THEMIS observations (Mozer et al., 2008; Bonnell et al., 2008). Contrary to the situation of a quadrupole Hall $\boldsymbol{B}_{M}$ and a bipolar Hall $\boldsymbol{E}_{N}$ in a symmetric reconnection, there is only one bump in the Hall field in the asymmetric reconnection. The observed asymmetric Hall electric fields and magnetic fields indicate the operation of collisionless reconnection.

The length of the diffusion region along the normal direction is the crossing time $\Delta T$ multiplied by the magnetopause velocity $v_{n}$ relative to the spacecraft. We estimate $v_{n}$ by assuming that the magnetopause had a constant tangential electric field as it moved in the normal direction at a constant speed. The estimated $v_{n}$ is $\left(\mathrm{E}_{M}(1)\right.$ $\left.\mathrm{E}_{M}(2)\right) /\left(\mathrm{B}_{L}[1]-\mathrm{B}_{L}[2]\right)$ (Mozer et al., 2002). Here the fields $\mathrm{E}_{M}(1), \mathrm{E}_{M}(2), \mathrm{B}_{L}[1]$ and $\mathrm{B}_{L}[2]$ are measured at the spacecraft frame at times 1 and 2 upstream and downstream of the current sheet, respectively. As shown in Fig. 2d and g, $E_{M}(1)$ 


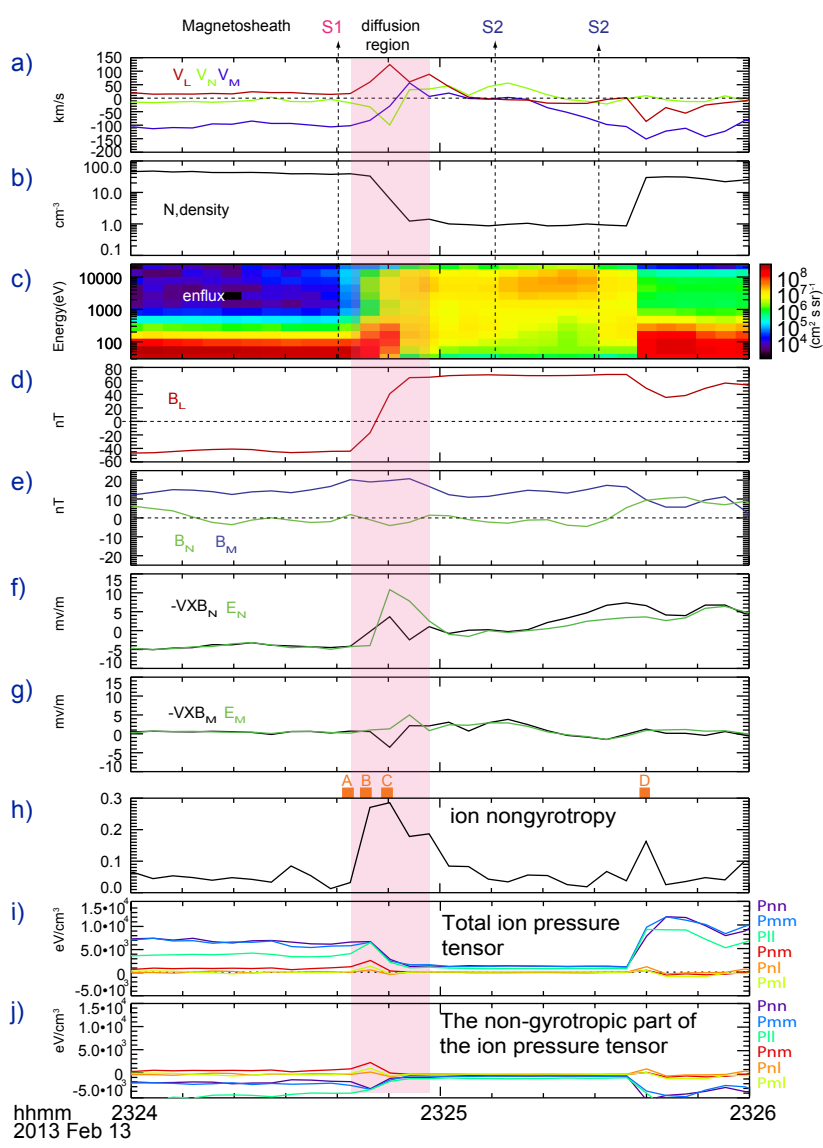

Figure 2. Observations by THEMIS spacecraft $\mathrm{E}$ in the ion diffusion region. All THEMIS data in Fig. 2 are of spin resolution and at the cadence of the particle instrument. Panel (a): three components of the proton flow velocity in the LMN coordinate system, showing a flow reversal in the $\mathbf{L}$ (north-south direction). Panel (b): the plasmas density. Panel (c): the electron differential energy flux. Panels (d) and (e): three component of magnetic field in the LMN coordinate. Panels (f) and (g): the comparison of $\boldsymbol{v}_{\mathbf{i}} \times \boldsymbol{B}$ with the electric fields in $\mathbf{N}$ and $\mathbf{M}$, respectively. Three-dimensional electric fields are obtained from the $\boldsymbol{E} \cdot \boldsymbol{B}=0$ assumption. Panel (h): the ion non-gyrotropy index (Aunai et al., 2013), characterizing the degree of non-gyrotropy in the distribution function. The ion velocity distribution function in intervals marked by A, B, C and D are show in Fig. 3. Panel (i): components of the ion pressure tensor in the boundary normal coordinate. Panel $(\mathbf{j})$ : the non-gyrotropic part of the ion pressure tensor.

is $\sim 0.5 \mathrm{mV} \mathrm{m}^{-1}$ and $\mathrm{B}_{L}[1]$ is $\sim-45 \mathrm{nT}$ from $23: 24: 20 \mathrm{UT}$ to $23: 24: 40 \mathrm{UT}$ before the crossing. Immediately after the crossing $\mathrm{E}_{M}(2)$ is $\sim 2.5 \mathrm{mV} \mathrm{m}^{-1}$ and $\mathrm{B}_{L}[2]$ is $\sim 70 \mathrm{nT}$ from 23:25:02 to $23: 25: 14 \mathrm{UT}$. The magnetopause velocity $\mathrm{V}_{n}$ is $\sim 17.4 \mathrm{~km} \mathrm{~s}^{-1}$. The length of the diffusion region along the normal direction is $264.5 \mathrm{~km}$, about 7.3 magnetosheath ion skin depths or 1.2 magnetosphere ion skin depths $\left(c / \omega_{p i}\right)$. This scale size is consistent with the values of the diffusion region in previously reported examples of collisionless reconnection (Mozer et al., 2002; Vaivads et al., 2004; Wygant et al., 2005). The estimated tangential reconnection electric field near the reversal of $\boldsymbol{B}_{L}$ is $\sim 0.5-1.3 \mathrm{mV}$ in the frame co-moving with the magnetopause current sheet.

The departure of the pressure tensor from cylindrical symmetry about the local magnetic field direction can be measured by a non-gyrotropy index (Aunai et al., 2013).The nongyrotropy and similar agyrotropy indexes have been successfully applied to characterize non-gyrotropic electrons in the electron diffusion region (Scudder et al., 2012; Tang et al., 2013; Aunai et al., 2013). The comparison between nongyrotropy and nonideal electric field, however, has not been done yet in these studies. In our event, Fig. $2 \mathrm{~h}$ shows an intensified layer of ion non-gyrotropy within the diffusion region, indicating strongly non-gyrotropic ions when the ions are demagnetized. The ion non-gyrotropy is as large as 0.3 in the diffusion region, significantly larger than the average value $(\sim 0.05)$ outside the diffusion region. Both the off-diagonal pressure component and the difference between the diagonal component can contribute to the non-gyrotropy index (Aunai et al., 2013). We find that the contribution from the offdiagonal pressure component is dominant $(>90 \%)$ in the diffusion region.

The non-gyrotropic pressure can lead to the violation of the ion (or electron) frozen-in condition via the off-diagonal terms in the pressure tensor. Similar to the situation of non-gyrotropic electron pressure (Vasyliunas, 1975; Hesse et al., 1999, 2011), the gradient of the off-diagonal ion pressure components can give rise to a nonideal reconnection electric field in the diffusion region in 2-D reconnection, $\left(\boldsymbol{E}+\boldsymbol{v}_{\mathrm{i}} \times \boldsymbol{B}\right)_{M} \sim\left(\partial P_{N M} / \partial N+\partial P_{M L} / \partial L\right) / n_{\mathrm{i}} q_{\mathrm{i}}$. Figure $2 \mathrm{i}$ presents the ion pressure tensor components in the boundary normal coordinate system. Spin-resolution ion moment data has been extensively used in the Walen test (or tangential component test) (Sonnerup et al., 1981). Ion moments data are generally considered acceptable when the spacecraft takes multiple samples in the reconnection current layer (Hoshino et al., 1998; Nagai et al., 1998; Øieroset et al., 2001; Mozer et al., 2002; Wygant et al., 2005; Zhou et al., 2009; Aunai et al., 2011). From 23:24:46 to 23:24:54 UT, THEMIS observed a significant nonideal reconnection electric field $\left(\boldsymbol{E}+\boldsymbol{v}_{\mathrm{i}} \times \boldsymbol{B}\right)_{M}$ of $3-4.5 \mathrm{mV} \mathrm{m}^{-1}$ (Fig. $\left.2 \mathrm{~g}\right)$ in the center of the diffusion region associated with a gradient of the ion off-diagonal pressure component (Fig. 2i). The density $n_{\mathrm{i}}$ (Fig. 2b) was $\sim 6.2 \mathrm{~cm}^{-3}$ at 23:24:50 UT, $\Delta N$ from 23:24:46 to 23:24:54 UT is $\sim-129 \mathrm{~km}, \mathrm{P}_{N M}$ (Fig. 2i) decreases from $2500 \mathrm{eV} \mathrm{cm}^{-3}$ to near 0 , and $\Delta P_{N M} \sim-2500 \mathrm{eV} \mathrm{cm}^{-3}$. With all these numbers, our estimate of $\Delta P_{N M} / \Delta N n_{\mathrm{i}} q_{\mathrm{i}}$ is $+3.2 \mathrm{mV} \mathrm{m}^{-1} . \Delta P_{M L}$ is $\sim-1000 \mathrm{eV} \mathrm{cm}^{-3}$ in Fig. 2 i. Assuming $\Delta L \sim \Delta N, \Delta P_{M L} / \Delta L n_{\mathrm{i}} q_{\mathrm{i}}$ is $\sim 1.3 \mathrm{mV} \mathrm{m}^{-1}$. The assumption of $\Delta L \sim \Delta N$ corresponds to an ion diffusion region extending 16 ion skin depth (roughly from 23:24:46 to 23:25:20 UT) northward in the $\mathbf{L}$ direction. Such an $L$-extent of the ion diffusion region is consistent with reconnection models. The gradient of the off-diagonal ion pressure terms agrees well with the nonideal reconnection electric field, in- 
a)

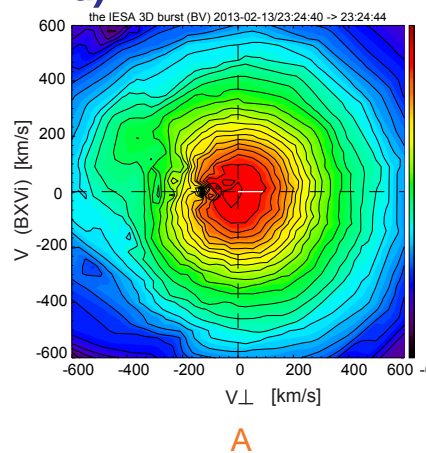

b)

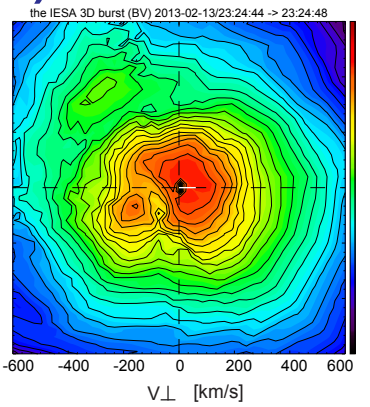

c)

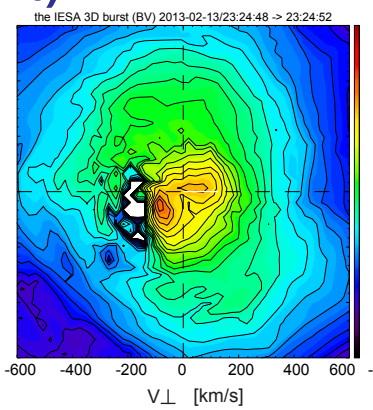

d)

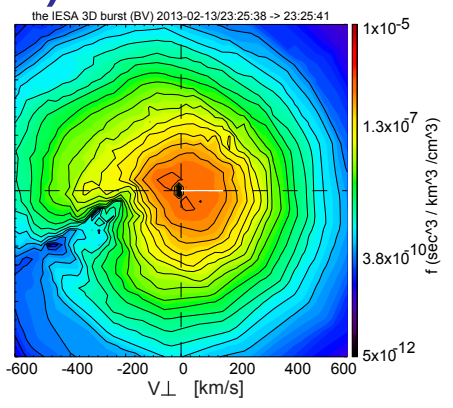

C

Figure 3. Observations of ion velocity distributions near and within the ion diffusion region. Panels (a-d): the cut of velocity distribution functions perpendicular to the magnetic field measured from THEMIS ESA in the intervals marked as A, B, C, and D in Fig. 2. The horizontal axis is the direction of ion convection flow $\boldsymbol{V}_{\mathrm{i} \perp}$ and the vertical axis is the direction of $\boldsymbol{B} \times \boldsymbol{V}_{\mathrm{i} \perp}$. The ion bulk flow velocity has been subtracted from the distributions. The white horizontal line in the distribution represents the flow convection velocity in the spacecraft frame. Interval A is far from the diffusion region, B and C are within the diffusion region and D is at the edge of the current layer and away from the current sheet center.

dicating that the non-gyrotropic pressure effect mainly contributed to breaking the ion frozen-in condition. Ion inertial (acceleration) effects and, in principle, the anomalous collision effect can also contribute to reconnection electric fields. In the diffusion region, $V_{N} \sim-70 \mathrm{~km} \mathrm{~s}^{-1}$ and $\Delta V_{M} \sim 130 \mathrm{~km} \mathrm{~s}^{-1}$ (Fig. 2a). The ion inertial term scales as $\left(m_{\mathrm{i}} / q_{\mathrm{i}}\right) V_{N} \Delta V_{M} / \Delta N\left(<0.3 \mathrm{mV} \mathrm{m}^{-1}\right)$, much less than the non-gyrotropic pressure effect. The anomalous collision term is expected to be very small because the observed reconnection should be collisionless, as implied by current sheet thickness (ion skin depth) and the Hall fields. The ion pressure tensor can be decomposed into a gyrotropic part and a non-gyrotropic part (Aunai et al., 2013). As shown in Fig. 2j, the non-gyrotropic part of the pressure tensor mostly contributes to the off-diagonal components. Based on the analysis of all possible effects in the ion momentum equation, we demonstrate that the non-gyrotropic pressure effect is primarily responsible for breaking the ion frozen-in condition in this event.

The ion momentum equation can be checked term by term in the normal direction as well. $\left(\boldsymbol{E}+\boldsymbol{v}_{\mathrm{i}} \times \boldsymbol{B}\right)_{N}$ is about $7-10 \mathrm{mV} \mathrm{m}^{-1}$ near the reversal of $\boldsymbol{B}_{L}$. The ion inertial term in the normal direction is on the order of $0.1 \mathrm{mV} \mathrm{m}^{-1}$. The gradient of the pressure term in the normal direction is $\left(\partial P_{N N} / \partial N+\partial P_{L N} / \partial L\right) / n_{\mathrm{i}} q_{\mathrm{i}}$. The $\Delta P_{N N} \sim-6000 \mathrm{eV} \mathrm{cm}^{-3}$ is the dominant term, contributing $7.8 \mathrm{mV} \mathrm{m}^{-1}$ in the ion momentum equation. Within a difference of $20 \%$, the pressure gradient term is approximately consistent with the $\left(\boldsymbol{E}+\boldsymbol{v}_{\mathrm{i}} \times \boldsymbol{B}\right)_{N}$. This independent verification of the ion momentum equation in the normal direction involves the quantitative comparison between three independent data sets, indicating that THEMIS measurements were reasonably reliable in the ion diffusion region. Notice that a significant uncertainty in the ion moment data may arise due to the time-aliasing effect, i.e., the mixing of different populations during the one spacecraft spin, but the level of the verification of the ion momentum equation in both $\mathbf{N}$ and $\mathbf{M}$ directions is very difficult to explain by arbitrary timealiasing effects.

As implied by the two-fluid momentum Eq. (1), electrons are expected to exhibit similar dynamics to ions except that the nonideal electron terms operate on the smaller electron scale in the diffusion region. Theoretical studies show that non-gyrotropic pressure effects unfreeze electron fluid near the reconnection $X$-line (Hesse et al., 1999; Kuznetsova et al., 2001). Encompassing the observation of ion nongyrotropic pressure, an emerging scenario is that the nongyrotropic pressure effect applies universally to unfrozen ions on a large spatial scale and to electrons on a small spatial scale. Near the $X$-line, the reconnection electric field should be equally expressed in terms of ion or electron nongyrotropic pressure (Hesse et al., 2011).

Figure 3 shows the ion velocity distributions perpendicular to the magnetic field near and in the diffusion region. Panel (a) shows a typical gyrotropic ion distribution in the magnetosheath outside the diffusion region. The distributions in panels (b) and (c) are centered around $\boldsymbol{B}_{L}=-20 \mathrm{nT}$ and $\boldsymbol{B}_{L}=+40 \mathrm{nT}$, where the magnetic field are found to deviate from the spin-averaged values by $\sim 30$ and $\sim 20 \%$ during one spin period. The velocity distributions in panels (b) and (c) are in the clearly identified ion diffusion region and characterized by non-gyrotropic bulges in the core component. According to the definition of the pressure tensor, $P=m \int \mathrm{d} v^{3}(\boldsymbol{v}-\boldsymbol{u})(\boldsymbol{v}-\boldsymbol{u}) f(\mathbf{x}, \boldsymbol{v}, t)$, where $\boldsymbol{u}$ is the bulk flow velocity, such non-gyrotropic bulges are expected to contribute to non-zero integral of $\left(\boldsymbol{v}_{M}-\boldsymbol{u}_{M}\right)\left(\boldsymbol{v}_{N}-\boldsymbol{u}_{N}\right)$ and thus to large off-diagonal pressure components. Two bulges are centered at $(50,15)$ and $(-82,-50) \mathrm{km} \mathrm{s}^{-1}$ in the interval $\mathrm{C}$. The velocity spread of these bulges is $\sim 100 \mathrm{~km} \mathrm{~s}^{-1}$, about half of the thermal speed in the diffusion region. The ob- 
served non-gyrotropic distribution is particularly important for magnetic field line reconfiguration in the diffusion region as it can drive a current sheet instability that induces the normal magnetic field component (Motschmann and Glassmeier, 1998a). In addition, the non-gyrotropic distributions are expected to drive plasma waves that tend to stabilize the plasma (Motschmann and Glassmeier, 1998b). The strongly non-Maxwellian distribution observed in the diffusion region has two implications: (1) the effective ion-electron collision is insufficient to lead to Maxwellian distributions while ions are demagnetized, and (2) the ion demagnetization process is much faster than ion thermalization and thus unlikely to be explained by effective ion-electron collisions. The nongyrotropic bulges in panels (b) and (c) resemble those reported by GEOTAIL spacecraft in reconnection (Hoshino et al., 1998; Nagai et al., 1998; Wygant et al., 2005). Numerical simulations suggest that non-gyrotropic bulges are caused by the mixing of already accelerated meandering ions with ions just convected into the vicinity of the $X$-line geometry (Hoshino et al., 1998; Hoshino, 1998). Such a mixing process is inferred to also occur in the asymmetric reconnection at the magnetopause from observations from Cluster (Lee et al., 2014). Ions from distinct sources form phasebunched bulges in the distribution. This scenario of forming non-gyrotropic bulges is supported by the difference between the energy of the non-gyrotropic bulges shown in panels (b) and (c). The non-gyrotropy in the warmer ion components in panel (d) resembles those in THEMIS observations outside the current sheet center but near the reconnection site (Zhou et al., 2009). These non-gyrotropic warmer ions are formed by a cucumber-type trajectory that has the neutral sheet-crossing and the non-crossing segments near an $X$-line geometry (Zhou et al., 2009).

\section{Summary and conclusions}

Ion thermal pressure effects have been neglected and the ion inertial effect is difficult to identity in the generalized Ohm's law. Therefore, the individual ion momentum equation without approximation is the best choice for investigating the ion-scale physics in reconnection. We take this approach and present clear evidence that a non-gyrotropic ion pressure situation is responsible for breaking the ion frozen-in condition in reconnection. For the first time, we show that the nonideal reconnection electric field associated with the violation of the ion frozen-in condition is consistent with the gradient of the off-diagonal ion pressure components. Our approach and results would be valuable for the MMS (Magnetospheric Multiscale Mission) that is designed to resolve all scales relevant to magnetic reconnection.

Electrons are expected to exhibit similar dynamics to ions except that the nonideal effect begins on the smaller electron scale. An emerging scenario is that the non-gyrotropic pressure effect universally unfreezes ions on the ion scale and electrons on a smaller scale in the collisionless reconnection. As is evident from the two-fluid momentum equation, the reconnection electric field is equally explainable in terms of ions or electrons. Near the $X$-line, the reconnection electric field may be equally expressed in terms of ion or electron non-gyrotropic pressure effect (Cai and Lee, 1997; Hesse et al., 2011).

Acknowledgements. THEMIS Data are available at http://themis. ssl.berkeley.edu. We thank the THEMIS and ancillary data providers. L. Dai thanks Steve Monson for proofreading the manuscript. We thank Judy Hohl for editorial help. K.-H. Glassmeier was financially supported through the German Ministry for Economy and Energy and the German Center for Aviation and Space (DLR) under contract 50 OC 0302. This work was supported by NASA contract NAS5-02099, NNSFC grant 41231067, 41574161 and in part by the Specialized Research Fund for State Key Laboratories of China.

The topical editor C. Owen thanks one anonymous referee for help in evaluating this paper.

\section{References}

Angelopoulos, V.: The THEMIS Mission, Space Sci. Rev., 141, 534, doi:10.1007/s11214-008-9336-1, 2008.

Angelopoulos, V., McFadden, J. P., Larson, D., Carlson, C. W., Mende, S. B., Frey, H., Phan, T., Sibeck, D. G., Glassmeier, K.-H., Auster, U., Donovan, E., Mann, I. R., Rae, I. J., Russell, C. T., Runov, A., Zhou, X.-Z., and Kepko, L.: Tail Reconnection Triggering Substorm Onset, Science, 321, 931-935, doi:10.1126/science.1160495, 2008.

Angelopoulos, V., Runov, A., Zhou, X.-Z., Turner, D. L., Kiehas, S. A., Li, S.-S., and Shinohara, I.: Electromagnetic Energy Conversion at Reconnection Fronts, Science, 341, 1478-1482, doi:10.1126/science.1236992, 2013.

Aunai, N., Retinò, A., Belmont, G., Smets, R., Lavraud, B., and Vaivads, A.: The proton pressure tensor as a new proxy of the proton decoupling region in collisionless magnetic reconnection, Ann. Geophys., 29, 1571-1579, doi:10.5194/angeo-291571-2011, 2011.

Aunai, N., Hesse, M., and Kuznetsova, M.: Electron nongyrotropy in the context of collisionless magnetic reconnection, Phys. Plasmas, 20, 092903, doi:10.1063/1.4820953, 2013.

Bonnell, J. W., Mozer, F. S., Delory, G. T., Hull, A. J., Ergun, R. E., Cully, C. M., Angelopoulos, V., and Harvey, P. R.: The Electric Field Instrument (EFI) for THEMIS, Space Sci. Rev., 141, 303341, doi:10.1007/s11214-008-9469-2, 2008.

Cai, H. J. and Lee, L. C.: The generalized Ohm's law in collisionless magnetic reconnection, Phys. Plasmas, 4, 509-520, doi:10.1063/1.872178, 1997.

Dai, L.: Collisionless Magnetic Reconnection via Alfvén Eigenmodes, Phys. Rev. Lett., 102, 245003, doi:10.1103/PhysRevLett.102.245003, 2009.

Dai, L., Wygant, J. R., Cattell, C., Dombeck, J., Thaller, S., Mouikis, C., Balogh, A., and Rème, H.: Cluster observations of surface waves in the ion jets from magne- 
totail reconnection, J. Geophys. Res.-Space, 116, A12227, doi:10.1029/2011JA017004, 2011.

Deng, X. H. and Matsumoto, H.: Rapid magnetic reconnection in the Earth's magnetosphere mediated by whistler waves, Nature, 410, 557-560, 2001.

Henderson, P. D., Owen, C. J., Lahiff, A. D., Alexeev, I. V., Fazakerley, A. N., Lucek, E., and Rème, H.: Cluster PEACE observations of electron pressure tensor divergence in the magnetotail, Geophys. Res. Lett., 33, L22106, doi:10.1029/2006GL027868, 2006.

Hesse, M., Schindler, K., Birn, J., and Kuznetsova, M.: The diffusion region in collisionless magnetic reconnection, Phys. Plasmas, 6, 1781-1795, doi:10.1063/1.873436, 1999.

Hesse, M., Neukirch, T., Schindler, K., Kuznetsova, M., and Zenitani, S.: The Diffusion Region in Collisionless Magnetic Reconnection, Space Sci. Rev., 160, 3-23, doi:10.1007/s11214-0109740-1, 2011.

Hoshino, M.: Kinetic Ion Behavior in Magnetic Reconnection Region, Washington D.C. American Geophysical Union Geophysical Monograph Series, 105, 153, 1998.

Hoshino, M., Mukai, T., Yamamoto, T., and Kokubun, S.: Ion dynamics in magnetic reconnection: Comparison between numerical simulation and Geotail observations, J. Geophys. Res., 103, 4509-4530, doi:10.1029/97JA01785, 1998.

Kuznetsova, M. M., Hesse, M., and Winske, D.: Collisionless reconnection supported by nongyrotropic pressure effects in hybrid and particle simulations, J. Geophys. Res, 106, 3799-3810, doi:10.1029/1999JA001003, 2001

Lee, S. H., Zhang, H., Zong, Q.-G., Otto, A., Sibeck, D. G., Wang, Y., Glassmeier, K.-H., Daly, P. W., and Rème, H.: Plasma and energetic particle behaviors during asymmetric magnetic reconnection at the magnetopause, J. Geophys. Res.-Space, 119, 16581672, doi:10.1002/2013JA019168, 2014.

McFadden, J. P., Carlson, C. W., Larson, D., Ludlam, M., Abiad, R., Elliott, B., Turin, P., Marckwordt, M., and Angelopoulos, V.: The THEMIS ESA Plasma Instrument and In-flight Calibration, Space Sci. Rev., 141, 277-302, doi:10.1007/s11214-008-9440-2, 2008.

Motschmann, U. and Glassmeier, K. H.: Relation of Magnetic Field Line Reconnection and Unstable Nongyrotropic Particle Distributions, in: Substorms-4, Astrophysics and Space Science Library, vol. 238, edited by: Kokubun, S. and Kamide, Y., p. 491, 1998a.

Motschmann, U. and Glassmeier, K.-H.: Dispersion and wave excitation in nongyrotropic plasmas, J. Plasma Phys., 60, 111-132, doi:10.1017/S002237789800676X, 1998b.

Mozer, F. S., Bale, S. D., and Phan, T. D.: Evidence of diffusion regions at a subsolar magnetopause crossing, Phys. Rev. Lett., 89, 015002, doi:10.1103/PhysRevLett.89.015002, 2002.

Mozer, F. S., Angelopoulos, V., Bonnell, J., Glassmeier, K. H., and McFadden, J. P.: THEMIS observations of modified Hall fields in asymmetric magnetic field reconnection, Geophys. Res. Lett., 35, L17S04, doi:10.1029/2007GL033033, 2008.

Nagai, T., Fujimot, M., Saito, Y., Machida, S., Terasawa, T., Nakamura, R., Yamamoto, T., Mukai, T., Nishida, A., and Kokubun, S.: Structure and dynamics of magnetic reconnection for substorm onsets with Geotail observations, J. Geophys. Res., 103, 4419-4440, doi:10.1029/97JA02190, 1998.
Nagai, T., Shinohara, I., Fujimoto, M., Hoshino, M., Saito, Y., Machida, S., and Mukai, T.: Geotail observations of the Hall current system: Evidence of magnetic reconnection in the magnetotail, J. Geophys. Res., 106, 25929-25950, doi:10.1029/2001JA900038, 2001.

Øieroset, M., Phan, T. D., Fujimoto, M., Lin, R. P., and Lepping, R. P.: In situ detection of collisionless reconnection in the Earth's magnetotail, Nature, 412, 414-417, 2001.

Parker, E. N.: Sweet's Mechanism for Merging Magnetic Fields in Conducting Fluids, J. Geophys. Res., 62, 509-520, 1957.

Petschek, H. E.: Magnetic Field Annihilation, in: The Physics of Solar Flares, edited by: Hess, W. N., p. 425, 1964.

Scudder, J. D., Holdaway, R. D., Daughton, W. S., Karimabadi, H., Roytershteyn, V., Russell, C. T., and Lopez, J. Y.: First Resolved Observations of the Demagnetized Electron-Diffusion Region of an Astrophysical Magnetic-Reconnection Site, Phys. Rev. Lett., 108, 225005, doi:10.1103/PhysRevLett.108.225005, 2012.

Sibeck, D. G. and Angelopoulos, V.: THEMIS Science Objectives and Mission Phases, Space Sci. Rev., 141, 35-59, doi:10.1007/s11214-008-9393-5, 2008.

Sonnerup, B. U. Ö.: Magnetic field reconnection, in: Solar System Plasma Physics, edited by: Lanzerotti, L. J., Kennel, C. F., and Parker, E. N., 45-108, 1979.

Sonnerup, B. U. Ö., Paschmann, G., Papamastorakis, I., Sckopke, N., Haerendel, G., Bame, S. J., Asbridge, J. R., Gosling, J. T., and Russell, C. T.: Evidence for magnetic field reconnection at the earth's magnetopause, J. Geophys. Res., 86, 10049-10067, doi:10.1029/JA086iA12p10049, 1981.

Tang, X., Cattell, C., Dombeck, J., Dai, L., Wilson, L. B., Breneman, A., and Hupach, A.: THEMIS observations of the magnetopause electron diffusion region: Large amplitude waves and heated electrons, Geophys. Res. Lett., 40, 2884-2890, doi:10.1002/grl.50565, 2013.

Vaivads, A., Khotyaintsev, Y., André, M., Retinò, A., Buchert, S. C., Rogers, B. N., Décréau, P., Paschmann, G., and Phan, T. D.: Structure of the magnetic reconnection diffusion region from four-spacecraft observations, Phys. Rev. Lett., 93, 105001, doi:10.1103/PhysRevLett.93.105001, 2004.

Vasyliunas, V. M.: Theoretical models of magnetic field line merging, Rev. Geophys. Space GE, 13, 303-336, 1975.

Wygant, J. R., Cattell, C. A., Lysak, R., Song, Y., Dombeck, J., McFadden, J., Mozer, F. S., Carlson, C. W., Parks, G., Lucek, E. A., Balogh, A., Andre, M., Reme, H., Hesse, M., and Mouikis, C.: Cluster observations of an intense normal component of the electric field at a thin reconnecting current sheet in the tail and its role in the shock-like acceleration of the ion fluid into the separatrix region, J. Geophys. Res.-Space, 110, A09206, doi:10.1029/2004JA010708, 2005.

Zhou, X.-Z., Angelopoulos, V., Runov, A., Sitnov, M. I., Zong, Q.-G., and Pu, Z. Y.: Ion distributions near the reconnection sites: Comparison between simulations and THEMIS observations, J. Geophys. Res.-Space, 114, A12211, doi:10.1029/2009JA014614, 2009. 